TRANSACTIONS OF THE

AMERICAN MATHEMATICAL SOCIETY

Volume 351, Number 10, Pages 3935-3952

S 0002-9947(99)02250-3

Article electronically published on February 8, 1999

\title{
THE SPACE OF COMPLETE MINIMAL SURFACES WITH FINITE TOTAL CURVATURE AS LAGRANGIAN SUBMANIFOLD
}

\author{
JOAQUÍN PÉREZ AND ANTONIO ROS
}

\begin{abstract}
The space $\mathcal{M}$ of nondegenerate, properly embedded minimal surfaces in $\mathbb{R}^{3}$ with finite total curvature and fixed topology is an analytic lagrangian submanifold of $\mathbb{C}^{n}$, where $n$ is the number of ends of the surface. In this paper we give two expressions, one integral and the other pointwise, for the second fundamental form of this submanifold. We also consider the compact boundary case, and we show that the space of stable nonflat minimal annuli that bound a fixed convex curve in a horizontal plane, having a horizontal end of finite total curvature, is a locally convex curve in the plane $\mathbb{C}$.
\end{abstract}

\section{INTRODUCTION}

The class of complete minimal surfaces in three-dimensional Euclidean space $\mathbb{R}^{3}$ is an important object of study in classical differential geometry. Within this field it is also natural to focus on the class of complete embedded surfaces with finite total curvature. Until recently, only a few examples in this class were known, but since the pioneering works of Costa, Hoffman and Meeks $[1,6,7]$ we have witnessed the birth of a large variety of examples, see for instance [2, 5, 10, 21]. Nowadays the understanding of the nature of this class as a whole constitutes an active field of research. A readable survey about this field is Hoffman and Karcher [5].

Properly embedded minimal surfaces in $\mathbb{R}^{3}$ have finite topology and their behaviour at infinity, i.e. at their ends, is controlled by a finite set of parameters; in fact, each surface of this type is asymptotic to a finite family of parallel planes and halfcatenoids. A similar phenomenon appears in the study of properly embedded (nonminimal) constant mean curvature surfaces in $\mathbb{R}^{3}$, see Korevaar, Kusner and Solomon [11], where the ends are also asymptotic to the revolution models. Such considerations led to the conjecture that the shape of the surface at infinity, that is, the geometric position and scale of these objects at infinity, should be natural parameters for the space of surfaces under consideration. This conjecture was confirmed in papers by Kusner, Mazzeo and Pollack [12] in the nonminimal case and by the authors [17] in the minimal setting. It should also be mentioned that the geometric principle at the heart of this conjecture has also been exploited in Kapouleas construction of examples in both families [8, 9]. An interesting feature of these developments is that only half of the potential configurations of ends can be realized by the ends of such a surface; the space of these surfaces forms a lagrangian

Received by the editors April 10, 1997.

1991 Mathematics Subject Classification. Primary 53A10, 53C42.

Research partially supported by a DGYCYT Grant No. PB94-0796.

(C)1999 American Mathematical Society 
submanifold (under suitable nondegeneracy assumptions) in a natural symplectic manifold, which in the case under consideration in this paper is simply $\mathbb{C}^{n}$ with its standard Kaehler form, where $n$ is the number of ends of the surface.

More precisely, in [17] the authors endowed the space of properly immersed minimal surfaces of finite total curvature, fixed topology and $n$ embedded parallel ends with a natural structure of finite dimensional real analytic manifold provided that a nondegeneracy condition is satisfied (this condition is defined in terms of the space of bounded Jacobi functions on the minimal surface). The tangent space at a nondegenerate surface $M$ is described as the linear space $\mathcal{J}(M)$ of Jacobi functions on $M$ having logarithmic singularities at the ends. The space $\mathcal{J}(M)$ is the kernel of the linearization $L$ (often called the Jacobi operator) of the mean curvature operator on $M$ acting on the space of allowed perturbations of $M$. Moreover, if $\mathcal{M}^{*}$ denotes the space of nondegenerate surfaces, then there exists a lagrangian immersion $f$ : $\mathcal{M}^{*} \longrightarrow \mathbb{C}^{n}$ defined by means of data depending only on the behaviour of the surfaces at infinity. The real and imaginary parts in each component of $f$ correspond to the scale (or logarithmic growth) and height of one of the ends of the minimal surface. A natural question is to investigate the geometry of such a submanifold.

The main goal of this paper is to give explicit expressions for the second fundamental form of the immersion above. Thanks to the lagrangian character of $f$, its second fundamental form at $M \in \mathcal{M}^{*}$ can be determined by a symmetric realvalued three-tensor $C_{M}(u, v, w)$, where $u, v, w \in \mathcal{J}(M)$. We will obtain (theorems 2 and 3) two expressions for $C_{M}(u, v, w)$. The first one involves information about $M, u, v, w$ along the whole surface,

$$
C_{M}(u, v, w)=\frac{1}{\pi} \int_{M}\{u \sigma(\nabla v, \nabla w)+w \sigma(\nabla u, \nabla v)+v \sigma(\nabla w, \nabla u)\} d A,
$$

where $\sigma$ denotes the (real valued) second fundamental form of $M \hookrightarrow \mathbb{R}^{3}$.

The second expression depends only on data of the surface and its Jacobi functions at the set of ends $F$ and at the branch points $B$ of its Gauss map (the dependence on $F$ was naturally expected, but that on $B$ is a little bit surprising):

$$
C_{M}(u, v, w)=4 \sum_{p \in B \cup F} \operatorname{Real}\left[\operatorname{Residue}_{p}\left(\frac{\partial u \partial v \partial w}{\omega}\right)\right],
$$

where $\omega$ is the Hopf quadratic differential of $M$ and $\partial u$ stands for the complex one-form $\frac{\partial u}{\partial z} d z$. It turns out that both $\partial u$ and $\frac{\partial u \partial v \partial w}{\omega}$ are complex one-forms with singularities exactly at $B \cup F$ and that those singularities have meromorphic principal part. Thus the residue of such a one-form at a point of $B \cup F$ is understood as the residue of its principal part. In the simplest situations it is possible to have a further control on this second fundamental form. For instance (see corollary 1),

If all the ends of $M \in \mathcal{M}^{*}$ are of catenoid type and all the branch points of its Gauss map have branching order equal to one, then given $u, v, w \in \mathcal{J}(M)$,

$$
C(u, v, w)=4 \sum_{p \in B} \frac{(\nabla \sigma)(\nabla u, \nabla v, \nabla w)}{\|\nabla \sigma\|^{2}}(p)+\sum_{p_{i} \in F} \frac{\log (u)_{i} \log (v)_{i} \log (w)_{i}}{\log (M)_{i}} .
$$

Here $\log (u)_{i}, \log (M)_{i}$ mean the $i$-th components of the lists of logarithmic growths of the Jacobi function $u$ and of the surface $M$ at the ends, respectively, and $\nabla \sigma$ denotes the covariant derivative of the second fundamental form of $M$ in $\mathbb{R}^{3}$. 
We will also study in Section 5 properly embedded minimal surfaces with finite total curvature having fixed topology and fixed (nonempty, compact) boundary. We first show that any stable surface $M$ is nondegenerate provided that it is noncompact (in the compact case this could be false). So, the space of its nearby minimal surfaces is an $n$-dimensional manifold, $n$ being the number of ends of the stable surface.

Finally, we consider the space $\mathcal{S}$ of stable minimal annuli bounded by a fixed convex curve in a horizontal plane, with a horizontal end of catenoid type. This space is one-dimensional, and the lagrangian submanifold is merely a planar curve. We will prove that this curve is locally convex. In [18] we study this space globally, showing for instance that the curve $\mathcal{S} \subset \mathbb{R}^{2}$ is embedded and has two connected components, given by the sign of the logarithmic growth of the end.

B. Palmer pointed out to us the strong parallel between our second fundamental form and the "Intrinsic third derivatives" of Tromba in [20]. It should be mentioned that the lagrangian structure appears, besides the minimal and the constant mean curvature cases explained above, when other moduli spaces of noncompact geometric objects are considered, see the works of Hauswirth, Kusner, Mazzeo, Pérez, Pollack and Uhlenbeck $[4,12,13,14,16,19]$. The arguments in the present paper could be useful in the study of the local geometry of those lagrangian submanifolds.

The authors wish to thank the referee for his comments.

\section{BACKGROUND}

We begin by recalling some basic facts that will be used in later sections. For details, see [17]. Let $\mathcal{M}=\mathcal{M}(k, n)$ be the space of all properly immersed minimal surfaces in $\mathbb{R}^{3}$ with finite total curvature, horizontal embedded ends and fixed topology (to avoid trivial cases, we will assume genus $k \geq 1$ and $n$ ends, $n \geq 3$ ), endowed with the uniform topology on compact sets of $\mathbb{R}^{3}$. Each $M \in \mathcal{M}$ is conformally a finitely punctured compact Riemann surface $\bar{M}$ and the punctures correspond to the ends of $M$. An end of $M$ is always a graph outside a disk in the plane $\left\{x_{3}=0\right\}$. Hence, after an inversion in this plane (which we will identify with the complex line $\mathbb{C}$ ), the end can be parametrized as

$$
\psi(w)=\left(\frac{1}{w},-a \log \rho+h(w)\right) \in \mathbb{C} \times \mathbb{R} \equiv \mathbb{R}^{3}, \quad 0<|w|<\varepsilon .
$$

Here, $w$ is a (nonconformal) coordinate of the compactified surface $\bar{M}$ around the end, $\rho=|w|, a$ is the logarithmic growth of the end, and $h$ is a smooth function in the whole disk $\{|w|<\varepsilon\}$. This lets us define the lists $\log (M) \in \mathbb{R}^{n}$ of logarithmic growths of the ends of $M$ and height $(M) \in \mathbb{R}^{n}$, this last one being the $n$-tuple whose components are the values $h(0)$ (called the height of the end) of the smooth functions appearing in (1) for each end. At a catenoid type end, the logarithmic growth coincides with the one of the asymptotic halfcatenoid and the height differs from the third coordinate of the center of this halfcatenoid in the term $a \log \left|\frac{a}{2}\right|$, while at a planar end the logarithmic growth is zero and the height coincides with the height of the asymptotic plane.

The Gauss map $N$ of $M$ can be written as

$$
N=\left\langle N, e_{3}\right\rangle\left(-a \bar{w}+\bar{w}^{2} \nabla_{0} h, 1\right),
$$

where $e_{3}=(0,0,1)$ and $\nabla_{0}$ is the gradient with respect to the flat metric in the $w$ plane. Note that $\left\langle N, e_{3}\right\rangle$ is a $C^{\infty}$ function taking values \pm 1 at the ends. Moreover, 
its gradient $\nabla_{0}\left\langle N, e_{3}\right\rangle$ has the form $\nabla_{0}\left\langle N, e_{3}\right\rangle=A(w) w+\rho^{2} X(w)$, where $A, X$ are smooth functions on $\{|w|<\varepsilon\}, A$ (resp. $X$ ) being real valued (resp. complex valued).

If $z$ is a conformal coordinate around one end, the change of parameters between $z$ and the coordinate $w$ in (1) is as $z=w t(w)$ (or $z=\bar{w} t(w)$ ), where $t$ is a complex valued smooth function with $t(0) \neq 0$.

The set of infinitesimal deformations of $M \in \mathcal{M}$ in this space is identified with the linear space of Jacobi functions, i.e. solutions of the equation

$$
\Delta u+\|\nabla N\|^{2} u=0 \quad \text { on } M,
$$

such that in the coordinate $w$ around each end $p_{i}, 1 \leq i \leq n$, are given by

$$
u(w)=-a_{i}\left\langle N, e_{3}\right\rangle \log \rho+u_{i}(w),
$$

with $a_{i} \in \mathbb{R}$ and $u_{i} \in C^{\infty}(\{|w|<\varepsilon\})$. Note that in a conformal coordinate $z$, the change of parameters between $z$ and $w$ insures that $u(z)$ has the form $u(z)=$ $-a_{i}\left\langle N, e_{3}\right\rangle \log |z|+\widetilde{u}_{i}(z)$ with $\widetilde{u}_{i}$ smooth at the origin.

The space $\mathcal{J}(M)$ is always finite-dimensional and contains the subspace $\mathcal{K}(M)$ of bounded Jacobi functions. In fact, the dimension of $\mathcal{J}(M)$ is given by $\operatorname{dim} \mathcal{J}(M)=$ $n+\operatorname{dim} \mathcal{K}_{0}(M)$, this last space being the set of Jacobi functions that vanish at all the ends. As isometries of $\mathbb{R}^{3}$ induce Jacobi functions on our surface, we always have three independent functions in $\mathcal{K}_{0}(M)$, namely $\left\langle N, e_{1}\right\rangle,\left\langle N, e_{2}\right\rangle, \operatorname{det}\left(p, N, e_{3}\right)$, hence $\operatorname{dim} \mathcal{J}(M) \geq n+3$. The surface $M$ is said to be nondegenerate if equality holds. For the class $\mathcal{M}^{*} \subset \mathcal{M}$ of nondegenerate minimal surfaces the following structure theorem holds.

Theorem 1 ([17]). $\mathcal{M}^{*}$ is an open subset of $\mathcal{M}$. If $\mathcal{M}^{*}$ is nonvoid, then it has a natural structure of an $(n+3)$-dimensional real analytic manifold, and the tangent space at a nondegenerate surface $M$ is given by $\mathcal{J}(M)$.

Fix a nondegenerate surface $M \in \mathcal{M}^{*}$. Given a function $u \in \mathcal{J}(M)$, we write

$$
\begin{aligned}
\log (u) & =\left(a_{1}, \ldots, a_{n}\right), \\
\operatorname{Height}(u) & =\left(u_{1}(0)\left\langle N\left(p_{1}\right), e_{3}\right\rangle, \ldots, u_{n}(0)\left\langle N\left(p_{n}\right), e_{3}\right\rangle\right) .
\end{aligned}
$$

These linear operators on $\mathcal{J}(M)$ and the log and height maps defined above have the following relationship. The map $f: \mathcal{M}^{*} \longrightarrow \mathbb{C}^{n}$ given by $f(M)=$ $(\log (M)$, height $(M))$ is real analytic and its differential is

$$
d f_{M}(u)=(\log (u), \operatorname{Height}(u)), \quad \forall u \in \mathcal{J}(M) .
$$

Moreover, if $\mathcal{G}$ denotes the three-dimensional Lie group generated by the horizontal translations and the rotations around the $x_{3}$-axis, then $\mathcal{G}$ acts analytically on $\mathcal{M}^{*}$ by means of $(\phi, M) \longmapsto \phi(M),(\phi, M) \in \mathcal{G} \times \mathcal{M}^{*}$, the quotient $\mathcal{M}^{*} / \mathcal{G}$ is a real analytic manifold of dimension $n$ and $f$ factorizes through this quotient, giving rise to a lagrangian immersion of $\mathcal{M}^{*} / \mathcal{G}$ into $\mathbb{C}^{n}$ with respect to the canonical symplectic structure.

In this paper we compute explicitly the second fundamental form of the lagrangian immersion $f: \mathcal{M}^{*} / \mathcal{G} \longrightarrow \mathbb{C}^{n}, f(M)=(\log (M)$, height $(M))$. As the Jacobi operator can be thought of as the linearization of the equation $H=0(H$ denotes mean curvature), this computation will involve the first derivative of the Jacobi operator for a deformation of a minimal immersion. For the remainder of the paper, we will denote the derivative of an expression $E_{t}(x)$ at $t=0$ by a dot, $\dot{E}(x)$. 
Proposition 1. Let $\psi_{t}: M \longrightarrow \mathbb{R}^{3},|t|<t_{0}$, be a smooth deformation of an orientable minimal immersion $\psi_{0}=\psi$, with variational field $\dot{\psi}=\dot{\psi}^{\top}+\dot{\psi}^{\perp}=\dot{\psi}^{\top}+\varphi N$, $N$ being the Gauss map of $\psi$ and $\varphi \in C^{\infty}(M, \mathbb{R})$, where $v^{\top}, v^{\perp}$ mean the tangential and normal parts of a vector $v$, respectively (note that $\psi_{t}$ is not assumed to be minimal for $t \neq 0)$. Consider the differential operator $L_{t}=\Delta_{t}+\left\|\sigma_{t}\right\|_{t}^{2}$ given by the laplacian in the induced metric plus the square length of the second fundamental form of $\psi_{t}$ (in particular, $L_{0}=L$ is the Jacobi operator of $\psi$ ). Then, for any function $u$ satisfying $L u=0$ on $M$ we have

$$
\left.\frac{d}{d t}\right|_{t=0} L_{t} u=2 \operatorname{div}(\varphi A \nabla u)+2 \operatorname{div}(A \nabla \varphi) u-L\left(d u\left(\dot{\psi}^{\top}\right)\right),
$$

where $A$ denotes the shape operator of $\psi$ and where $\nabla$, div stand for the gradient and the divergence operators, respectively.

Proof. As the derivative is a linear operator, we can study separately the cases $\dot{\psi}=\varphi N$ and $\dot{\psi}=\dot{\psi}^{\top}$.

In the first case, we will need expressions of the first variation of some geometrical objects on $M$. Given a function $f \in C^{2}(M, \mathbb{R})$ and a tangent vector field $X$ on $M$, we have that the first variation formulas for the induced metric $g$, the gradient operator $\nabla$, the normal vector $N$, the divergence operator div, the laplacian operator $\Delta$, the second fundamental form $\sigma$ and its square length $\|\sigma\|^{2}$ are respectively given by

$$
\begin{aligned}
\dot{g} & =-2 \varphi \sigma, \\
\dot{\nabla} f & =2 \varphi A \nabla f, \\
\dot{N} & =-d \psi(\nabla \varphi), \\
\overbrace{\operatorname{div} X}=0, & \\
\dot{\Delta} f & =2 \operatorname{div}(\varphi A \nabla f), \\
\dot{\sigma} & =\nabla^{2} \varphi-\frac{1}{2}\|\sigma\|^{2} \varphi g, \\
\overbrace{\|\sigma\|^{2}}^{2} & =2 \operatorname{div}(A \nabla \varphi),
\end{aligned}
$$

where $\nabla^{2}$ denotes the Hessian operator. These formulas can be obtained easily. We will prove only the last four.

To compute $\overbrace{\operatorname{div}} X$, differentiate with respect to $t$ the expression $L_{X} d A_{t}=$ $\operatorname{div}_{t} X d A_{t}$, where $L_{X}$ stands for the usual Lie derivative and use that $\left.\frac{d}{d t}\right|_{t=0} d A_{t}=$ 0 because $\psi$ is minimal and $\dot{\psi}$ is normal. As $\Delta f=\operatorname{div}(\nabla f)$, the computation of $\dot{\Delta} f$ follows from the former formulas.

In order to obtain $\dot{\sigma}$, we differentiate the equation

$$
\sigma_{t}(X, Y)=-\left\langle d N_{t}(X), d \psi_{t}(Y)\right\rangle,
$$

where $N_{t}$ is the Gauss map of $\psi_{t}$ and $X, Y$ are tangent vectors to $M$. Thus,

$$
\begin{aligned}
\dot{\sigma}(X, Y) & =-\langle d \dot{N}(X), d \psi(Y)\rangle-\langle d N(X), d \dot{\psi}(Y)\rangle \\
& =g\left(\nabla_{X} \nabla \varphi, Y\right)-g(A X, \varphi A Y) \\
& =\left(\nabla^{2} \varphi\right)(X, Y)-\frac{1}{2}\|\sigma\|^{2} \varphi g(X, Y),
\end{aligned}
$$

where we have used that, for a minimal surface, $A^{2}$ is proportional to the identity. 
Let us compute now the derivative of $\left\|\sigma_{t}\right\|_{t}^{2}$. Given an orthonormal basis $e_{1}, e_{2}$ of the tangent space at a point $p \in M$, we get

$$
\begin{gathered}
\left.\frac{d}{d t}\right|_{t=0}\left\|\sigma_{t}\right\|_{t}^{2}=2 \sum_{i, j} \sigma\left(e_{i}, e_{j}\right) \dot{\sigma}\left(e_{i}, e_{j}\right)-2 \sum_{i, j, k} \sigma\left(e_{i}, e_{j}\right) \sigma\left(e_{j}, e_{k}\right) \dot{g}\left(e_{i}, e_{k}\right) \\
=2 \sum_{i, j} \sigma\left(e_{i}, e_{j}\right)\left(\nabla^{2} \varphi\right)\left(e_{i}, e_{j}\right)=2 \operatorname{div}(A \nabla \varphi),
\end{gathered}
$$

where we have used in the last equality that the contraction of the covariant derivative of $A$ vanishes because of the minimality of $\psi$. This proves the proposition when $\dot{\psi}$ is normal.

Suppose now that $\dot{\psi}=\dot{\psi}^{\top}$. As $\dot{L}$ does not depend on the deformation but only on the variational field, we can take $\psi_{t}$ as $\psi \circ \phi_{t}$, where $\phi_{t}$ is the local one-parameter flow associated to the tangent field $\dot{\psi}^{\top}$. Thus $L_{t}\left(u \circ \phi_{t}\right)=(L u) \circ \phi_{t}=0$ because $u$ is a Jacobi function. Differentiating this equation at $t=0$ we have $\dot{L} u+L d u(\dot{\psi})=0$, and the proposition is proved in the second case.

For later uses, we will need the first variation of the Gauss map in the general case, $\dot{\psi}^{\top} \neq 0$. Given a tangent vector $x$ to $M$, differentiating the equality $\left\langle N_{t}, d \psi_{t}(x)\right\rangle=0$ gives $0=\langle\dot{N}, d \psi(x)\rangle+\sigma\left(\dot{\psi}^{\top}, x\right)+\langle d \psi(\nabla \varphi), d \psi(x)\rangle$. As $\langle\dot{N}, N\rangle=0$, we deduce

$$
\dot{N}=-d \psi\left(A \dot{\psi}^{\top}+\nabla \varphi\right) .
$$

We will also need a slight generalization of Lemma 5.1 in [17]. As the proof is similar to the case studied there, we will not include it here.

Lemma 1. For a (possibly degenerate) surface $M \in \mathcal{M}$, consider two functions $u, v \in C^{2}(M)$ with expressions of the type

$$
u(w)=-a_{i}(w) \log |w|+u_{i}(w), \quad v(w)=-b_{i}(w) \log |w|+v_{i}(w)
$$

with respect to the coordinate $0<|w|<\varepsilon$ for each end $p_{i}$ of $M, i=1, \ldots, n$, where $a_{i}, b_{i}, u_{i}, v_{i} \in C^{2}(\{|w|<\varepsilon\})$. Then, $u L v$ is integrable on $M$ and

$$
\int_{M}(u L v-v L u) d A=2 \pi \sum_{i=1}^{n}\left(b_{i}(0) u_{i}(0)-a_{i}(0) v_{i}(0)\right) .
$$

\section{The SECOND FUndamental FORM: The INTEGRAL EXPRESSION}

Consider on $\mathbb{R}^{2 n}=\mathbb{C}^{n}$ the canonical symplectic two-form

$$
\Omega\left((\mathbf{a}, \mathbf{b}),\left(\mathbf{a}^{\prime}, \mathbf{b}^{\prime}\right)\right)=\left\langle\mathbf{a}, \mathbf{b}^{\prime}\right\rangle-\left\langle\mathbf{a}^{\prime}, \mathbf{b}\right\rangle, \quad(\mathbf{a}, \mathbf{b}),\left(\mathbf{a}^{\prime}, \mathbf{b}^{\prime}\right) \in \mathbb{R}^{n} \times \mathbb{R}^{n}=\mathbb{R}^{2 n} .
$$

The second fundamental form $\sigma_{f}: T \mathcal{N} \times T \mathcal{N} \longrightarrow T \mathcal{N}^{\perp}$ of a lagrangian immersion $f: \mathcal{N}^{n} \longrightarrow \mathbb{R}^{2 n}, \mathcal{N}^{n}$ being a smooth $n$-dimensional manifold, can be given in a more natural way by means of a real valued three-tensor as follows:

$$
C(X, Y, Z)=\Omega\left(\sigma_{f}(X, Y), Z\right)=\Omega(X(Y(f)), Z(f)),
$$

$X, Y, Z$ being tangent vector fields to $\mathcal{N}$.

Differentiating the lagrangian condition $\Omega(X(f), Y(f))=0$ with respect to $Z \in$ $T \mathcal{N}$ we conclude the well-known fact that $C$ is symmetric in its three arguments. We are interested in finding an expression for this second fundamental form in the case $f: \mathcal{M}^{*} / \mathcal{G} \longrightarrow \mathbb{R}^{2 n}, \quad f([M])=(\log (M)$, height $(M))$. 
Take a nondegenerate surface $M \in \mathcal{M}^{*}$ and a Jacobi function $u \in \mathcal{J}(M)$. By the definition above and the symmetry of $C$, we can restrict ourselves to the expression

$$
C(u, u, u)=\Omega\left(\left.\frac{d}{d t}\right|_{0} d f_{\left[M_{t}\right]}\left(u_{t}\right), d f_{[M]}(u)\right),
$$

where $M_{t}$ is a curve in $\mathcal{M}^{*}$ passing through $M$ at $t=0$ with velocity vector $u$ and $u_{t} \in \mathcal{J}\left(M_{t}\right)$ is the tangent field to this curve.

We will represent the surfaces $M_{t}$ by means of a smooth family of immersions $\psi_{t}: M \longrightarrow \mathbb{R}^{3}$ such that in the coordinate $w$ around each end (see (1)),

$$
\psi_{t}(w)=\left(\frac{1}{w}, v_{t}(w)\right), \quad 0<|w|<\varepsilon
$$

where $v_{t}(w)$ is a function with a logarithmic singularity at $w=0$ that depends smoothly on $t$. From (3), we have that $u=\langle\dot{\psi}, N\rangle, u_{t}=\left\langle\frac{d}{d t} \psi_{t}, N_{t}\right\rangle$ are functions that satisfy the hypotheses of Lemma 1 as functions on both $M$ and $M_{t}$. Hence, using (5) for the surface $\psi_{t}: M \longrightarrow \mathbb{R}^{3}$ and that $L_{t} u_{t}=0$ we deduce

$$
\Omega\left(d f_{M_{t}}\left(u_{t}\right), d f_{M}(u)\right)=-\frac{1}{2 \pi} \int_{M} u_{t} L_{t} u d A_{t}
$$

where the subscript " $t$ " denotes that the corresponding object is computed with respect to the metric induced by $M_{t}$. By direct computation around the ends (using an auxiliary conformal coordinate), we find an expression of the type

$$
u_{t} L_{t} u d A_{t}=\left[A(t, w)(\log \rho)^{2}+B(t, w) \log \rho+C(t, w)\right] d A_{w},
$$

where $A, B, C$ are smooth in $t$ and in $w \in\{|w|<\varepsilon\}$ and $d A_{w}$ denotes the Euclidean measure in the $w$-plane. So, we can differentiate the right-hand side of the expression (7) under the integral sign. As $L u=0$, from (6) we have

Lemma 2. In the situation above,

$$
C(u, u, u)=-\frac{1}{2 \pi} \int_{M} u \dot{L} u d A
$$

Using Proposition 1 in formula (8) we obtain

$$
C(u, u, u)=-\frac{1}{\pi} \int_{M}\left(u \operatorname{div}(u A \nabla u)+u^{2} \operatorname{div}(A \nabla u)-\frac{1}{2} u L\left(d u\left(\dot{\psi}^{\top}\right)\right)\right) d A .
$$

We claim that the function $u L\left(d u\left(\dot{\psi}^{\top}\right)\right)$ is integrable on $M$ and that its integral vanishes. With this aim, note that by Lemma 1 we only have to prove that $d u\left(\dot{\psi}^{\top}\right)$ can be written around each end as $-b_{i}(w) \log |w|+v_{i}(w), b_{i}$ and $v_{i}$ being $C^{2}$ functions on $\{|w|<\varepsilon\}$ that vanish at $w=0$. So, let us consider an end of our surface, expressed in the $w$ coordinate as in (1). As $\langle\dot{\psi}, N\rangle=u$ and $\dot{\psi}=(0, \dot{v})$, we have $\dot{v}\left\langle N, e_{3}\right\rangle=u$, thus

$$
\dot{\psi}^{\top}=\dot{\psi}-u N=\frac{u}{\left\langle N, e_{3}\right\rangle} e_{3}-u N
$$

Given a tangent vector $Y=\left(Y_{\mathbb{C}}, Y_{3}\right) \in \mathbb{R}^{3}=\mathbb{C} \times \mathbb{R}$ to the surface $\psi(M)$, from (1) one has that the vector $Z$ in the $w$-plane that satisfies $d \psi(Z)=Y$ is given by $Z=-w^{2} Y_{\mathbb{C}}$, so if we take $Y$ as $\dot{\psi}^{\top}$, from (2) and (10) we will obtain

$$
Z=u w^{2}\left\langle N, e_{3}\right\rangle\left(-a \bar{w}+\bar{w}^{2} \nabla_{0} h\right)=u\left\langle N, e_{3}\right\rangle \rho^{2}\left(-a w+\rho^{2} \nabla_{0} h\right) .
$$


Here $\nabla_{0}$ represents the gradient operator with respect to the flat metric in the $w$-plane. Thus,

$$
d u\left(\dot{\psi}^{\top}\right)=\left\langle Z, \nabla_{0} u\right\rangle=\rho^{2} u\left\langle N, e_{3}\right\rangle\left[-a\left\langle w, \nabla_{0} u\right\rangle+\rho^{2}\left\langle\nabla_{0} h, \nabla_{0} u\right\rangle\right] .
$$

Using that $u$ has a logarithmic singularity and that $\nabla_{0}\left\langle N, e_{3}\right\rangle$ vanishes at $w=0$, it is straightforward to conclude that $\rho^{2}\left[-a\left\langle w, \nabla_{0} u\right\rangle+\rho^{2}\left\langle\nabla_{0} h, \nabla_{0} u\right\rangle\right]$ is a $C^{2}$ function on $\{|w|<\varepsilon\}$. As it vanishes at $w=0$, Lemma 1 applied to the functions $u$ and $d u\left(\dot{\psi}^{\top}\right)$ proves that $\int_{M} u L\left(d u\left(\dot{\psi}^{\top}\right)\right) d A=0$, as we claimed.

Now we state the main result of this section.

Theorem 2. Let $M$ be a nondegenerate surface. Then, the second fundamental form of the lagrangian immersion $f=(\log$, height $)$ at $[M] \in \mathcal{M}^{*} / \mathcal{G}$ is given by

$$
C_{[M]}([u],[v],[w])=\frac{1}{\pi} \int_{M}\{u \sigma(\nabla v, \nabla w)+w \sigma(\nabla u, \nabla v)+v \sigma(\nabla w, \nabla u)\} d A,
$$

for any $[u],[v],[w] \in \mathcal{J}(M) / \mathcal{K}_{0}(M), \sigma$ being the (real valued) second fundamental form of $M \hookrightarrow \mathbb{R}^{3}$.

Proof. First observe that $u \sigma(\nabla u, \nabla u)$ is $d A$-integrable. In fact, taking an auxiliary conformal coordinate $z,|z|<\varepsilon_{1}$, around an end, we have $u g(\nabla u, A \nabla u) d A=$ $u g_{1}\left(\nabla_{1} u, A \nabla_{1} u\right) d A_{1}$, where the subscript "1" means that the corresponding object is computed with respect to the Euclidean geometry of the $z$-plane. As the eigenvalues of $A$ go to zero at least as $|z|^{2}$ and $u$ has a logarithmic singularity, it follows that $u g_{1}\left(\nabla_{1} u, A \nabla_{1} u\right)$ grows at most logarithmically, so its integral is finite. In particular, the integral in the statement of our theorem exists. Now taking into account that

$$
\begin{aligned}
\operatorname{div}\left(u^{2} A \nabla u\right) & =u \operatorname{div}(u A \nabla u)+u g(\nabla u, A \nabla u) \\
& =u^{2} \operatorname{div}(A \nabla u)+2 u g(\nabla u, A \nabla u),
\end{aligned}
$$

equation (9) can be written as

$$
C(u, u, u)=\frac{1}{\pi} \int_{M}\left[-2 \operatorname{div}\left(u^{2} A \nabla u\right)+3 u g(\nabla u, A \nabla u)\right] d A .
$$

Now to prove the theorem it suffices to check that $\int_{M} \operatorname{div}\left(u^{2} A \nabla u\right) d A=0$. Let us call, for $\delta>0$ small enough, $M(\delta)$ to the original surface $M$ with a collection of disks $\{|z|<\delta$ \} removed, $z$ being a conformal coordinate around each end. Then, the divergence theorem gives

$$
\int_{M(\delta)} \operatorname{div}\left(u^{2} A \nabla u\right) d A=\int_{\partial M(\delta)} u^{2} g(A \nabla u, \eta) d s,
$$

$\eta$ being the unit exterior conormal field to $\psi$ along $\partial M(\delta)$. We can parametrize this boundary by $n$ disjoint copies of $z=r e^{i \theta}, \theta \in[0,2 \pi]$. As the expression $\eta d s$ is conformal invariant, we have $\eta d s=-\frac{\partial}{\partial r}|d z|$, and this implies

$$
\int_{|z|=\delta} u^{2} g(A \nabla u, \eta) d s=\int_{|z|=\delta} u^{2} d u(A \eta) d s=-\int_{|z|=\delta} u^{2} d u\left(A \frac{\partial}{\partial r}\right)|d z| .
$$

As the shape operator $A$ goes to zero at least with order $r^{2}$, it follows that $u^{2} d u\left(A \frac{\partial}{\partial r}\right)$ grows, at most, as $r(\log r)^{2}$; thus the integral above tends to zero when $\delta$ goes to zero. Now the proof is complete. 
Remark 1. As a consequence of (9) and (11), the second fundamental form is also given by

$$
C(u, u, u)=-\frac{3}{\pi} \int_{M} u \operatorname{div}(u A \nabla u) d A=-\frac{3}{2 \pi} \int_{M} u^{2} \operatorname{div}(A \nabla u) d A .
$$

\section{The Second Fundamental form: The pointwise EXPRession}

In this section we will obtain a second expression for $C(u, u, u)$ that only depends on the behaviour of $M$ and $u$ at the ends and at the finite branch points of the Gauss map. We will use a preliminary lemma.

Lemma 3. Let $u$ and $v$ be harmonic functions on $D^{*}=\{0<|z| \leq 1\}$ such that $u$ has at most a logarithmic singularity at $z=0$ and $v=\operatorname{Real}(m), m$ being holomorphic on $D^{*}$ with at most a pole at the origin. Then, for all $\left.\left.r \in\right] 0,1\right]$,

$$
\int_{|z|=r}\left(u \partial_{r} v-v \partial_{r} u\right)|d z|=-4 \pi \text { Real } \operatorname{Res}_{0}(m \partial u),
$$

where $\partial u=u_{z} d z$ and $\operatorname{Res}_{0}$ denotes the residue of the corresponding meromorphic differential.

Proof. First note that for a pair of holomorphic functions $F, G$ on $D=\{|z| \leq 1\}$ and $D^{*}$ respectively, $G$ with at most a pole at $z=0$, it can be deduced by expanding in power series that

$$
\int_{|z|=r} \frac{F}{z} \bar{G} d z=2 \pi i F(0) \overline{\operatorname{Res}_{0}\left(\frac{G}{z} d z\right)}+\mathcal{O}(r), \quad 0<r \leq 1 .
$$

On the other hand, $u \partial_{r} v-v \partial_{r} u=2 r^{-1}$ Real $\left[z\left(u v_{z}-u_{z} v\right)\right]$, hence

$$
\begin{aligned}
\int_{|z|=r}\left(u \partial_{r} v-v \partial_{r} u\right)|d z| & =2 \operatorname{Real} \int_{0}^{2 \pi} z\left(u v_{z}-u_{z} v\right) d \theta \\
& =2 \operatorname{Imag} \int_{|z|=r}\left(u v_{z}-u_{z} v\right) d z
\end{aligned}
$$

We will call $\beta=\operatorname{Imag}(\alpha)$, with $\alpha=\left(u v_{z}-u_{z} v\right) d z$. As $u, v$ are harmonic in $D^{*}$, we have $\partial_{\bar{z}}\left(u v_{z}-u_{z} v\right)=u_{\bar{z}} v_{z}-u_{z} v_{\bar{z}}=2 i \operatorname{Imag}\left(u_{\bar{z}} v_{z}\right)$, and therefore $d \alpha=$ $2 i \operatorname{Imag}\left(u_{\bar{z}} v_{z}\right) d \bar{z} \wedge d z$ is a real valued two-form. As $d \beta=\operatorname{Imag}(d \alpha)$, we conclude the closedness of $\beta$. On the other hand, developing the expression

$$
4 \alpha=\left[(2 a \log r+l+\bar{l}) m^{\prime}-\left(\frac{a}{z}+l^{\prime}\right)(m+\bar{m})\right] d z,
$$

where we have written $u=a \log r+\operatorname{Real}(l)$ with $a \in \mathbb{R}$ and $l$ a holomorphic function in $\{|z| \leq 1\}$, we obtain

$$
\begin{aligned}
4 \int_{|z|=r} \alpha= & 2 a \log r \int_{|z|=r} m^{\prime} d z-a \int_{|z|=r} \frac{m}{z} d z+\int_{|z|=r}\left(l m^{\prime}-l^{\prime} m\right) d z \\
& -a \int_{|z|=r} \frac{\bar{m}}{z} d z+\int_{|z|=r}\left(\bar{l} m^{\prime}-l^{\prime} \bar{m}\right) d z .
\end{aligned}
$$

The first integral in the expression above vanishes, while the second one gives $2 \pi i \operatorname{Res}\left(\frac{m}{z} d z\right)$. After integration by parts, the third one yields $-4 \pi i \operatorname{Res}\left(l^{\prime} m d z\right)$. 
The last two terms can be treated with (13), giving

$$
\begin{aligned}
\int_{|z|=r} \frac{\bar{m}}{z} d z & =2 \pi i \overline{\operatorname{Res}_{0}\left(\frac{m}{z} d z\right)}+\mathcal{O}(r), \\
\int_{|z|=r}\left(\bar{l} m^{\prime}-l^{\prime} \bar{m}\right) d z & =\mathcal{O}(r) .
\end{aligned}
$$

Therefore,

$$
4 \int_{|z|=r} \alpha=-4 \pi i\left\{a \text { Real }\left[\operatorname{Res}_{0}\left(\frac{m}{z} d z\right)\right]+\operatorname{Res}_{0}\left(l^{\prime} m d z\right)\right\}+\mathcal{O}(r) .
$$

This implies

$$
\begin{aligned}
4 \int_{|z|=r} \beta & =-4 \pi\left\{a \operatorname{Real}\left[\operatorname{Res}_{0}\left(\frac{m}{z} d z\right)\right]+\operatorname{Real}\left[\operatorname{Res}_{0}\left(l^{\prime} m d z\right)\right]\right\}+\mathcal{O}(r) \\
& =-4 \pi \operatorname{Real}\left[\operatorname{Res}_{0}\left(\left(\frac{a}{z}+l^{\prime}\right) m d z\right)\right]+\mathcal{O}(r) .
\end{aligned}
$$

As we can eliminate the term $\mathcal{O}(r)$ in the last expression because $\beta$ is closed, the lemma is proved.

As the Gauss map $N$ of a minimal surface is (anti)meromorphic, in a neighbourhood of each $p \in M$ where $N$ is unbranched we can reparametrize the family of surfaces $\psi_{t}$ as a new family $\phi_{t}$ of conformal minimal immersions with fixed Gauss map $N$. An important consequence of these properties is that the Jacobi operator $L_{t}$ differs from $L$ by a positive function $\Lambda_{t}, L_{t}=\Lambda_{t} L$ in the neighbourhood above. In particular, the Jacobi equation does not depend on $t$. Therefore, if $B(N) \subset M$ is the set of branch points of the Gauss map $N$ and $u$ is a Jacobi function, we can apply Proposition 1 to the deformation $\phi_{t}$, obtaining

$$
L\left(d u\left(\dot{\phi}^{\top}\right)\right)=2 \operatorname{div}(u A \nabla u)+2 \operatorname{div}(A \nabla u) u \quad \text { in } \quad M-B(N) .
$$

Moreover, if the variational field is $\dot{\phi}=\dot{\phi}^{\top}+u N$, using the first variation of the Gauss map (4), we get $0=A \dot{\phi}^{\top}+\nabla u$ and thus $\dot{\phi}^{\top}=\frac{-2}{\|\sigma\|^{2}} A \nabla u$. The function $\varphi=$ $-\frac{1}{2} d u\left(\dot{\phi}^{\top}\right)=\frac{1}{\|\sigma\|^{2}} \sigma(\nabla u, \nabla u)$ can be also expressed in terms of the Hopf quadratic meromorphic differential $\omega=\sigma\left(\partial_{z}, \partial_{z}\right)(d z)^{2}=-\frac{1}{2} g^{\prime} f(d z)^{2}$, where $(g, f d z)$ are the Weierstrass data of $M$ with respect to a local conformal coordinate $z$ as follows

$$
\varphi=-2 \text { Real } \frac{\left(u_{z}\right)^{2}}{g^{\prime} f}=\operatorname{Real} \frac{(\partial u)^{2}}{\omega}
$$

where $\partial u=u_{z} d z$.

Let $\Omega(\varepsilon)$ be a small neighbourhood of the set $B(N) \cup F(M)$ in the compactification $\bar{M}$, where $F(M)=\left\{p_{1}, \ldots, p_{n}\right\}$ is the set of ends of $M$. Using (9) we deduce that

$$
\begin{aligned}
C(u, u, u) & =-\frac{1}{\pi} \lim _{\varepsilon \rightarrow 0} \int_{M-\Omega(\varepsilon)}\left(u \operatorname{div}(u A \nabla u)+u^{2} \operatorname{div}(A \nabla u)\right) d A \\
& =\frac{1}{\pi} \lim _{\varepsilon \rightarrow 0} \int_{M-\Omega(\varepsilon)} u L \varphi d A=\frac{1}{\pi} \lim _{\varepsilon \rightarrow 0} \int_{M-\Omega(\varepsilon)}(u L \varphi-\varphi L u) d A .
\end{aligned}
$$

Now the divergence theorem gives

$$
C(u, u, u)=\frac{1}{\pi} \lim _{\varepsilon \rightarrow 0} \int_{\partial(M-\Omega(\varepsilon))}\left(u \frac{\partial \varphi}{\partial \eta}-\varphi \frac{\partial u}{\partial \eta}\right) d s,
$$


where $\eta$ denotes the exterior conormal unit field to $\psi$ along the boundary of $M-$ $\Omega(\varepsilon)$. If $z=r e^{i \theta}$ is a conformal coordinate around a point $p$ in $B(N) \cup F(M)$, such that $z=0$ corresponds to $p$, one concludes that

$$
C(u, u, u)=-\frac{1}{\pi} \sum_{B(N) \cup F(M)} \lim _{r \rightarrow 0} \int_{|z|=r}\left(u \partial_{r} \varphi-\varphi \partial_{r} u\right)|d z|,
$$

where $\partial_{r}$ stands for $\frac{\partial}{\partial r}$. Next we will compute the limit in (15) at a point $p \in$ $B(N) \cup F(M)$.

First case: At a finite branch point of the Gauss map. Let us suppose that $p \in M$ is a finite branch point. Without lost of generality, we can write $g(z)=g(0)+z^{k} g_{1}(z)$, where $k \geq 2$ is an integer and $g_{1}$ is a holomorphic function with $g_{1}(0) \neq 0$. The function $f$ that determines the Weierstrass data $(g, f d z)$ of $M$ is also holomorphic and $f(0) \neq 0$. In particular, the Hopf differential $\omega$ has a zero of order $k-1$ at $z=0$. We will use the notation $A_{z^{a} \bar{z}^{b}}$ for $\frac{\partial^{a+b} A}{\partial z^{a} \partial \bar{z}^{b}}$. As the Jacobi equation in the $z$-coordinate is

$$
u_{z \bar{z}}+\frac{2\left|g^{\prime}\right|^{2}}{\left(|g|^{2}+1\right)^{2}} u=0
$$

we deduce that

$$
u_{z^{\alpha} \bar{z}^{\beta}}(0)=0 \quad \text { if } \quad 1 \leq \alpha, 1 \leq \beta \text { and } \alpha+\beta<2 k .
$$

This gives $u=u_{\mathrm{H}}+\mathcal{O}\left(r^{2 k}\right)$, where $u_{\mathrm{H}}$ is harmonic in a neighbourhood of $z=0$. Hence

$$
\partial u=\partial u_{\mathrm{H}}+\mathcal{O}\left(r^{2 k-1}\right), \quad \frac{(\partial u)^{2}}{\omega}=\frac{\left(\partial u_{\mathrm{H}}\right)^{2}}{\omega}+\mathcal{O}\left(r^{k}\right)
$$

and so

$$
\varphi=\operatorname{Real} \frac{\left(\partial u_{\mathrm{H}}\right)^{2}}{\omega}+\mathcal{O}\left(r^{k}\right) .
$$

As $\frac{\left(\partial u_{\mathrm{H}}\right)^{2}}{\omega}$ has a pole of order at most $k-1$, although $u, \varphi$ are not harmonic, the part of our limit (15) that involves the nonharmonic terms vanishes. Thus the value of the integral we would like to compute coincides with the one obtained by replacing $u$ by $u_{\mathrm{H}}$ and $\varphi$ by Real $\frac{\left(\partial u_{\mathrm{H}}\right)^{2}}{\omega}$. Now Lemma 3 gives that

$$
\lim _{r \rightarrow 0} \int_{|z|=r}\left(u \partial_{r} \varphi-\varphi \partial_{r} u\right)|d z|=-4 \pi \operatorname{Real} \operatorname{Res}_{p} \frac{\left(\partial u_{\mathrm{H}}\right)^{3}}{\omega} .
$$

On the other hand, we can deduce from (17) that $\frac{(\partial u)^{3}}{\omega}=\frac{\left(\partial u_{\mathrm{H}}\right)^{3}}{\omega}+\mathcal{O}\left(r^{k}\right)$. Then, it makes sense to define the residue at $p$ of the one-form $\frac{(\partial u)^{3}}{\omega}$ as the residue at zero of $\frac{\left(\partial u_{\mathrm{H}}\right)^{3}}{\omega}$, so we can write

$$
\lim _{r \rightarrow 0} \int_{|z|=r}\left(u \partial_{r} \varphi-\varphi \partial_{r} u\right)|d z|=-4 \pi \text { Real } \operatorname{Res}_{p} \frac{(\partial u)^{3}}{\omega} .
$$


Second case: At an end of $M$. Now we assume that $p$ is an end. The Gauss map $g$ can be supposed to have a zero at $p$, so $g(z)=z^{k} g_{1}(z), k \geq 1$ and $g_{1}$ is as before. The embeddedness of the end of $M$ implies that $f$ has a double pole without residue at this point, $f=z^{-2}\left(a_{0}+z^{2} f_{1}\right)$, with $a_{0} \in \mathbb{C}-\{0\}$ and $f_{1}$ holomorphic. Thus $\omega$ has a zero of order $k-3$ at the origin. As $u$ can have a logarithmic singularity at the end, we can write

$$
\begin{aligned}
u & =-a\left\langle N, e_{3}\right\rangle \log r+u_{1} \\
& =-a \frac{|g|^{2}-1}{|g|^{2}+1} \log r+u_{1}=a \log r+u_{1}+\mathcal{O}\left(r^{2 k} \log r\right),
\end{aligned}
$$

where $a \in \mathbb{R}$ and $u_{1}$ is a real analytic function in a neighbourhood of $z=0$. Reasoning as above, (16) remains true, and as

$$
u_{z}=\frac{a}{2 z}+\left(u_{1}\right)_{z}+\mathcal{O}\left(r^{2 k-1} \log r\right)
$$

it follows that the same order derivatives for $u_{1}$ also vanish. This implies that

$$
u=u_{\mathrm{H}}+\mathcal{O}\left(r^{2 k} \log r\right),
$$

with $u_{\mathrm{H}}$ a harmonic function with at most a logarithmic singularity at $z=0$. Moreover,

$$
\partial u=\partial u_{\mathrm{H}}+\mathcal{O}\left(r^{2 k-1} \log r\right), \quad \frac{(\partial u)^{2}}{\omega}=\frac{\left(\partial u_{\mathrm{H}}\right)^{2}}{\omega}+\mathcal{O}\left(r^{k+1} \log r\right),
$$

and

$$
\varphi=\operatorname{Real} \frac{\left(\partial u_{\mathrm{H}}\right)^{2}}{\omega}+\mathcal{O}\left(r^{k+1} \log r\right) .
$$

As above, the part of the limit involving nonharmonic terms vanishes, and using Lemma 3 we obtain

$$
\lim _{r \rightarrow 0} \int_{|z|=r}\left(u \partial_{r} \varphi-\varphi \partial_{r} u\right)|d z|=-4 \pi \text { Real } \operatorname{Res}_{p} \frac{\left(\partial u_{\mathrm{H}}\right)^{3}}{\omega} .
$$

As in the first case, we can use the equalities in (18) in order to obtain that $\frac{(\partial u)^{3}}{\omega}$ differs from $\frac{\left(\partial u_{\mathrm{H}}\right)^{3}}{\omega}$ in a term which decays as $r$ goes to zero. Thus, the residue of this one-form makes sense and our limit becomes

$$
\lim _{r \rightarrow 0} \int_{|z|=r}\left(u \partial_{r} \varphi-\varphi \partial_{r} u\right)|d z|=-4 \pi \text { Real } \operatorname{Res}_{p} \frac{(\partial u)^{3}}{\omega} .
$$

In conclusion, the complex one-form $\frac{(\partial u)^{3}}{\omega}$ has singularities with meromorphic principal part on $B(N) \cup F(M)$ and it is smooth everywhere else on $M$. Moreover, (15) gives $C(u, u, u)=4 \operatorname{Real} \sum\left[\left(\operatorname{Res}_{p} \frac{(\partial u)^{3}}{\omega}\right)\right]$, where the sum runs through $p \in$ $B(N) \cup F(M)$. As $C$ is symmetric, we can state the following.

Theorem 3. Let $M \in \mathcal{M}^{*}$ be a nondegenerate minimal surface with Hopf differential $\omega$. Then, the second fundamental form of $f=\left(\log\right.$, height) at $[M] \in \mathcal{M}^{*} / \mathcal{G}$ is given by

$$
C_{[M]}([u],[v],[w])=4 \sum_{B(N) \cup F(M)} \operatorname{Real}\left[\operatorname{Res}_{p}\left(\frac{\partial u \partial v \partial w}{\omega}\right)\right],
$$

where $[u],[v],[w]$ are tangent vectors in $\mathcal{J}(M) / \mathcal{K}_{0}(M)$. 
Hence, each finite branch point or end $p$ of the surface contributes by a term

$$
C_{p}(u, v, w)=4 \operatorname{Real}\left[\operatorname{Res}_{p}\left(\frac{\partial u \partial v \partial w}{\omega}\right)\right]
$$

to the second fundamental form $C_{[M]}([u],[v],[w])$. In certain particular cases, we can find expressions for this term from other points of view, which can be helpful in distinct approaches to the problem of computing this second fundamental form.

1) Consider a small disk $D(\varepsilon)$ containing a finite branch point $p \in B(N)$. Then, $C_{p}(u, u, u)$ coincides with the limit

$$
C_{p}(u, u, u)=\frac{2}{\pi} \lim _{\varepsilon \rightarrow 0} \int_{\partial D(\varepsilon)} \frac{1}{\|\sigma\|^{2}} \sigma(\nabla u, \nabla u) \frac{\partial u}{\partial \eta} d s,
$$

where $\eta$ is the interior conormal and $d s$ the arclength of $\partial D(\varepsilon)$ in the induced metric.

To prove this equality, we will use the same notation given before the last theorem. First note that

$$
\begin{aligned}
\lim _{\varepsilon \rightarrow 0} \int_{\partial D(\varepsilon)} & \frac{1}{\|\sigma\|^{2}} \sigma(\nabla u, \nabla u) \frac{\partial u}{\partial \eta} d s \\
& =-2 \lim _{r \rightarrow 0} \int_{|z|=r} \operatorname{Real}\left(\frac{\left(u_{z}\right)^{2}}{g^{\prime} f}\right) * d u \\
& =-2 \lim _{r \rightarrow 0} \operatorname{Real} \int_{|z|=r}\left[\frac{\left(u_{z}\right)^{2}}{g^{\prime} f}\left(-i u_{z} d z+i u_{\bar{z}} d \bar{z}\right)\right]
\end{aligned}
$$

where we have used the Hodge star operator of $M$. The last expression is equal to

$$
-2 \lim _{r \rightarrow 0} \operatorname{Imag} \int_{|z|=r} \frac{\left(u_{z}\right)^{3}}{g^{\prime} f} d z+2 \lim _{r \rightarrow 0} \operatorname{Imag} \int_{|z|=r} \frac{\left(u_{z}\right)^{2}}{g^{\prime} f} u_{\bar{z}} d \bar{z} .
$$

The first limit is $-4 \pi \operatorname{Real}\left[\operatorname{Res}_{p}\left(\frac{\left(u_{z}\right)^{3}}{g^{\prime} f} d z\right)\right]$, while the second one vanishes by (13) (also note that the second limit does not vanish at an end of $M$ ). This proves (19) at a finite branch point $p \in B(N)$. In fact, the argument above can be repeated without changes if we consider simultaneously three Jacobi functions $u, v, w \in \mathcal{J}(M)$, appearing $\frac{1}{\|\sigma\|^{2}} \sigma(\nabla u, \nabla v) \frac{\partial w}{\partial \eta}$ in the integral of the right-hand side of (19).

2) If $p$ is a finite branch point of minimum branching order of the Gauss map, then

$$
C_{p}(u, u, u)=-8 \text { Real }\left[\frac{\left(u_{z}\right)^{3}}{g^{\prime \prime} f}(p)\right]=4 \frac{(\nabla \sigma)(\nabla u, \nabla u, \nabla u)}{\|\nabla \sigma\|^{2}}(p),
$$

where $\nabla \sigma$ denotes the covariant derivative of the second fundamental form of $M$.

To prove this formula, observe that the principal part of $\frac{(\partial u)^{3}}{\omega}$ has a simple pole, and the first equality above holds. To rewrite this expression in terms of the Riemannian geometry of $M$, recall that the gradients $\nabla_{0} u, \nabla u$ in the flat metric $|d z|^{2}$ on the $z$-plane and in the induced metric $d s^{2}=\lambda^{2}|d z|^{2}$ are related by $\nabla u=\lambda^{-2} \nabla_{0} u=2 \lambda^{-2}\left(u_{z} \partial_{\bar{z}}+u_{\bar{z}} \partial_{z}\right)$. This implies

$$
\begin{aligned}
(\nabla \sigma)(\nabla u, \nabla u, \nabla u)(p)= & 8 \lambda^{-6}\left[\left(u_{z}\right)^{3}(\nabla \sigma)\left(\partial_{\bar{z}}, \partial_{\bar{z}}, \partial_{\bar{z}}\right)\right. \\
& \left.+\left(u_{\bar{z}}\right)^{3}(\nabla \sigma)\left(\partial_{z}, \partial_{z}, \partial_{z}\right)\right](p) \\
= & 16 \lambda^{-6} \operatorname{Real}\left[\left(u_{\bar{z}}\right)^{3}(\nabla \sigma)\left(\partial_{z}, \partial_{z}, \partial_{z}\right)\right](p),
\end{aligned}
$$


where we have annihilated the terms $(\nabla \sigma)\left(\partial_{z}, \partial_{z}, \partial_{\bar{z}}\right)$ and $(\nabla \sigma)\left(\partial_{z}, \partial_{\bar{z}}, \partial_{\bar{z}}\right)$ because $M$ is minimal and $\sigma$ vanishes at $p$. Similarly we conclude that

$$
(\nabla \sigma)\left(\partial_{z}, \partial_{z}, \partial_{z}\right)(p)=\partial_{z} \sigma\left(\partial_{z}, \partial_{z}\right)=-\frac{1}{2} \partial_{z}\left(g^{\prime} f\right)(p)=-\frac{1}{2}\left(g^{\prime \prime} f\right)(p) .
$$

In particular, $\|\nabla \sigma\|^{2}(p)=4 \lambda^{-6}\left|g^{\prime \prime} f\right|^{2}(p)$ and the second equality above is proved. Remark 2. If $p$ is a single branch point of $N$, the branching value $N(p) \in \mathbb{S}^{2}$ persists along the deformation $M_{t}$ with tangent vector $u$, giving rise to a spherical curve $\gamma(t)$. As the shape operator vanishes at $p$, from the first variation of the Gauss map (4) we deduce that $\gamma^{\prime}(0)=-\nabla u(p)$. In particular, if the curve $\gamma(t)$ is constant, we conclude that $C_{p}(u, u, u)=0$.

3) Another interesting case is at a catenoid type end $p_{i}$, in which (18) implies that

$$
C_{p_{i}}(u, u, u)=\frac{\left(\log (u)_{i}\right)^{3}}{\log (M)_{i}}
$$

and the subscript stands for the $i$-th component of the list of logarithmic growths of $u$ or of $M$. Thus, we can give a more explicit expression for the second fundamental form when the branch points of the Gauss map are simple:

Corollary 1. If all the ends of $M \in \mathcal{M}^{*}$ are of catenoid type and all the branch points of its Gauss map have branching order equal to one, then given $u, v, w \in$ $\mathcal{J}(M)$

$$
C(u, v, w)=4 \sum_{p \in B(N)} \frac{(\nabla \sigma)(\nabla u, \nabla v, \nabla w)}{\|\nabla \sigma\|^{2}}(p)+\sum_{p_{i} \in F(M)} \frac{\log (u)_{i} \log (v)_{i} \log (w)_{i}}{\log (M)_{i}} .
$$

4) For a catenoid type end $p$ of $M$ with logarithmic growth $a$, the height of the center of the asymptotic halfcatenoid does not coincide with height $(p)$ but is given by height ${ }_{c}(p)=\operatorname{height}(p)+a \log \left|\frac{a}{2}\right|$. If we consider the open subset $\mathcal{M}_{c}^{*}$ of $\mathcal{M}^{*}$ consisting of all nondegenerate minimal surfaces with ends of catenoid type, then it is natural to consider, instead of the immersion $f$, the map $f_{c}: \mathcal{M}_{c}^{*} / \mathcal{G} \longrightarrow \mathbb{R}^{2 n}$ given by $f_{c}(M)=\left(\log (M)\right.$, height $\left.{ }_{c}(M)\right)$, where height h $_{c}(M) \in \mathbb{R}^{n}$ is the list whose components are the heights of the centers of the asymptotic catenoids to the ends of $M$ (note that $f_{c}$ does not extend smoothly to $\mathcal{M}^{*}$ ). For this map $f_{c}$, we have the following properties:

Corollary 2. The map $f_{c}: \mathcal{M}_{c}^{*} / \mathcal{G} \longrightarrow \mathbb{R}^{2 n}$ is a lagrangian immersion and its second fundamental form $C_{c}$ at a point $[M] \in \mathcal{M}_{c}^{*} / \mathcal{G}$ is given by

$$
C_{c}([u],[v],[w])=4 \sum_{p \in B(N)} \operatorname{Real}\left[\operatorname{Res}_{p}\left(\frac{\partial u \partial v \partial w}{\omega}\right)\right],
$$

where $u, v, w \in \mathcal{J}(M)$.

Observe that the terms corresponding to the ends of $M$ do not appear now. The statement above follows directly from Theorem 3, (20) and the next elementary lemma whose proof we do not include.

Lemma 4. Consider a lagrangian immersion $f: \mathcal{N}^{n} \longrightarrow \mathbb{R}^{n} \times \mathbb{R}^{n}, f=\left(a_{1}, \ldots, a_{n}\right.$, $\left.b_{1}, \ldots, b_{n}\right)$ of an $n$-dimensional manifold $\mathcal{N}$ into the Euclidean space. Take real valued functions of class $C^{2} H_{i}(t), 1 \leq i \leq n$, and define

$$
f^{*}=\left(a_{1}, \ldots, a_{n}, b_{1}+H_{1}\left(a_{1}\right), \ldots, b_{n}+H_{n}\left(a_{n}\right)\right) .
$$


Then, $f^{*}$ is again a lagrangian immersion, and the symmetric tensors $C$ and $C^{*}$ associated to the second fundamental form of $f$ and $f^{*}$ respectively, are related by the equation

$$
C^{*}(X, X, X)=C(X, X, X)-\sum_{i=1}^{n} \frac{d^{2} H_{i}}{d t^{2}}\left(a_{i}\right)\left[d a_{i}(X)\right]^{3},
$$

for any tangent vector $X$ to $\mathcal{N}$.

\section{Minimal SURFACES With FIXED BOUNDARY}

All our development in [17] and in the sections above extends almost without change to minimal surfaces with fixed compact boundary. Consider the space of properly immersed minimal surfaces in $\mathbb{R}^{3}$ with finite total curvature, fixed topology, $n$ embedded horizontal ends and boundary given by a finite number of smooth Jordan curves in $\mathbb{R}^{3}$, that do not depend on the surface. For the sake of simplicity, we will suppose that this boundary is not the exceptional case of a collection of coaxial circles in horizontal planes (although this case can be also studied with our methods, some differences appear between this exceptional case and the general situation). The allowed perturbations will fix the boundary and grow logarithmically at infinity, hence the space $\mathcal{J}(M)$ will consist of the Jacobi functions that vanish at the boundary and with logarithmic growth at the ends. Similarly as in the case without boundary (see section 5 in [17]), it can be proved that the dimension of $\mathcal{J}(M)$ is greater that or equal to $n$. Nondegenerate surfaces are defined as those in which equality holds, i.e. $\operatorname{dim} \mathcal{J}(M)=n$ or, equivalently, when the space of bounded Jacobi functions $\mathcal{K}_{0}(M)$ that vanish at the boundary and at all the ends reduces to zero. The structure theorem in this situation can be stated as follows:

Theorem 4. The space $\mathcal{M}^{*}$ of nondegenerate properly immersed minimal surfaces with finite total curvature, fixed topology, $n$ embedded horizontal ends and given nonexceptional boundary (that does not depend on the surface) is a real analytic manifold of dimension $n$. The tangent space at a surface $M \in \mathcal{M}^{*}$ coincides with the linear space $\mathcal{J}(M)$ of logarithmically growing Jacobi functions with zero boundary values.

Recall that a noncompact minimal surface $M$ with boundary is called stable if for each compact subdomain of $M$ and with respect to any nontrivial normal variation fixing its boundary, the second derivative of the area functional is nonnegative.

Let $M \subset \mathbb{R}^{3}$ be an orientable noncompact complete minimal surface, and assume that $M$ is stable. Under these hypotheses, $M$ has finite total curvature (see FischerColbrie [3]). Suppose that all the ends of $M$ are parallel (this condition holds, for instance, when $M$ is embedded). Stability also implies that the compactified Jacobi operator $\bar{L}=\lambda L$ (here $\lambda$ is a positive smooth function that grows as $|w|^{-4}$ when $|w|$ goes to zero, see [3] and [17], page 188) has nonnegative first eigenvalue $\lambda_{1}$. If $\lambda_{1}$ is positive (in this case the surface is often called strictly stable), the space $\mathcal{K}(M)$ of bounded Jacobi functions vanishing at the boundary is zero, hence $M$ is nondegenerate. When $\lambda_{1}=0$, then $\mathcal{K}(M)$ has dimension 1 and its elements have no interior zeroes. This means that $\mathcal{K}_{0}(M)=\{0\}$ and $M$ is again nondegenerate. Thus we have

Lemma 5. Any orientable noncompact complete stable minimal surface with compact boundary and parallel ends is nondegenerate. 
By combining the results above, we conclude

Corollary 3. The space of orientable noncompact complete strictly stable minimal surfaces with fixed (both) topology and nonexceptional boundary is a real analytic manifold whose dimension coincides with the number of ends of the surface.

The description of this manifold as a lagrangian submanifold of $\mathbb{C}^{n}$ is as follows: The map $f: \mathcal{M}^{*} \longrightarrow \mathbb{C}^{n}, f(M)=(\log (M)$, height $(M))$ is real analytic and the kernel of its differential at $M \in \mathcal{M}^{*}$ is $\mathcal{K}_{0}(M)=\{0\}$, thus $f$ is an immersion which can be proved to be lagrangian, like in the empty boundary case.

Concerning its second fundamental form, the only steps of the computation in Sections 3, 4 to have to take into account are the following:

1. The skew-symmetric formula (5) in Lemma 1 holds, but for a pair of functions $u, v$ that vanish identically on $\partial M$.

2. When we use the divergence theorem in the first integral of (12), no new terms on $\partial M$ appear because the Jacobi function $u$ vanishes at the boundary. As consequence, Theorem 2 remains true.

3. Concerning the pointwise expression, to avoid possible difficulties in the computation of the limit in (14), we will suppose that the Gauss map of $M$ is unbranched along the boundary $\partial M$. The divergence theorem in (14) produces a boundary term, namely $-\frac{1}{\pi} \int_{\partial M} \varphi \frac{\partial u}{\partial \eta} d s$, where $\eta$ is the exterior conormal to the surface along its boundary. This fact, together with the local computation of the limit in (15), allows us to write

Theorem 5. The second fundamental form of $f=(\log$, height $)$ at a surface $M \in$ $\mathcal{M}^{*}$ having no branch points of its Gauss map at the boundary is given by

$$
C_{M}(u, u, u)=4 \sum_{B(N) \cup F(M)} \operatorname{Real}\left[\operatorname{Res}_{p}\left(\frac{(\partial u)^{3}}{\omega}\right)\right]-\frac{1}{\pi} \int_{\partial M} \frac{\sigma(\nabla u, \nabla u)}{\|\sigma\|^{2}} \frac{\partial u}{\partial \eta} d s,
$$

where $u \in \mathcal{J}(M), \omega$ is the Hopf differential of $M$ and $\sigma$ is its second fundamental form.

If instead of $f$ we consider the immersion $f_{c}=\left(\log\right.$, height $\left.{ }_{c}\right): \mathcal{M}_{c}^{*} \longrightarrow \mathbb{C}^{n}$, where $\mathcal{M}_{c}^{*}$ is the open subset of $\mathcal{M}$ consisting of those surfaces only with catenoid type ends and height ${ }_{c}$ denotes the heights of the centers of the asymptotic halfcatenoids, then the second fundamental form $C_{c}$ of $f_{c}$ coincides with the one above, up to the terms at the ends:

$$
C_{c}(u, u, u)=4 \sum_{p \in B(N)} \operatorname{Real}\left[\operatorname{Res}_{p}\left(\frac{(\partial u)^{3}}{\omega}\right)\right]-\frac{1}{\pi} \int_{\partial M} \frac{\sigma(\nabla u, \nabla u)}{\|\sigma\|^{2}} \frac{\partial u}{\partial \eta} d s .
$$

In the following particular but interesting case, we can obtain a more precise geometric description of this lagrangian submanifold.

Corollary 4. Let $\Gamma$ be a convex Jordan curve in a horizontal plane. Call $\mathcal{S}$ the space of strictly stable properly embedded nonflat minimal annuli having $\Gamma$ as boundary and with a horizontal end. Then, $\mathcal{S}$ is a one-dimensional real analytic manifold and the immersed curve $f_{c}(\mathcal{S}) \subset \mathbb{R}^{2}$ by $f_{c}=\left(\log\right.$, height $\left.{ }_{c}\right)$ is locally strictly convex.

Proof. The smoothness of $\mathcal{S}$ follows from Corollary 3. Given $M \in \mathcal{S}$, the maximum principle for harmonic functions insures that its end is of catenoid type. From the 
convexity of $\Gamma$ it follows easily (see [15] or [18]) that the Gauss map of $M$ has no branch points either at the interior or at the boundary. Thus, given $u \in \mathcal{J}(M)-\{0\}$, we get from (21) that the second fundamental form of $f_{c}$ at $M$ is

$$
C_{c}(u, u, u)=-\frac{1}{\pi} \int_{\Gamma} \frac{\sigma(\nabla u, \nabla u)}{\|\sigma\|^{2}} \frac{\partial u}{\partial \eta} d s
$$

As $u$ vanishes along the boundary of $M$, the integrand above can be expressed as

$$
\frac{\sigma(\eta, \eta)}{\|\sigma\|^{2}}\left(\frac{\partial u}{\partial \eta}\right)^{3}=-\frac{\sigma\left(\gamma^{\prime}, \gamma^{\prime}\right)}{\|\sigma\|^{2}}\left(\frac{\partial u}{\partial \eta}\right)^{3}=-\kappa \frac{\langle n, N\rangle}{\|\sigma\|^{2}}\left(\frac{\partial u}{\partial \eta}\right)^{3}
$$

where $\gamma^{\prime}, n$ and $\kappa$ are respectively, the tangent vector, the unit normal vector and the curvature of the planar curve $\Gamma$ (note that we have used the minimality of $M$ in the first equality above). As $M$ is strictly stable, the Jacobi function $u$ has a nonzero logarithmic singularity at the end and does not change its sign at the interior of $M$, hence by the boundary maximum principle $\frac{\partial u}{\partial \eta}$ does not change its sign at $\Gamma$. This implies that the integrand in (22) has constant sign and $C(u, u, u)$ is nonzero, which is equivalent to our convexity assertion on the curve $f_{c}(\mathcal{S})$.

\section{REFERENCES}

1. C. Costa, Example of a complete minimal immersion in $\mathbb{R}^{3}$ of genus one and three embedded ends, Bull. Soc. Bras. Mat. 15 (1984) 47-54. MR 87c:53111

2. C. Costa, Classification of complete minimal surfaces in $\mathbb{R}^{3}$ with finite total curvature $12 \pi$, Invent. Math. 105 (1991) 273-303. MR 92h:53010

3. D. Fischer-Colbrie, On complete minimal surfaces with finite Morse index in three manifolds, Invent. Math. 82 (1985) 121-132. MR 87b:53090

4. L. Hauswirth, On periodic minimal surfaces of type Scherk, preprint.

5. D. Hoffman \& H. Karcher, Complete embedded minimal surfaces of finite total curvature, Springer, Berlin, 1997. CMP 98:06.

6. D. Hoffman \& W. H. Meeks III, A complete minimal surface in $\mathbb{R}^{3}$ with genus one and three ends, J. Differential Geometry 21 (1985) 109-127. MR 87d:53008

7. D. Hoffman \& W. H. Meeks III, Embedded minimal surfaces of finite topology, Ann. Math. 131 (1990) 1-34. MR 91i:53010

8. N. Kapouleas, Complete constant mean curvature surfaces in euclidean three space, Ann. Math. 131 (1990) 239-330. MR 93a:53007a

9. N. Kapouleas, Complete embedded minimal surfaces of finite total curvature, J. Differential Geom. 47 (1997), 95-169. CMP 98:07

10. H. Karcher, Embedded minimal surfaces derived from Scherk's examples, Manuscripta Math. 62 (1988) 83-114. MR 89i:53009

11. N. Korevaar, R. Kusner \& B. Solomon, The structure of complete embedded surfaces with constant mean curvature, J. Diff. Geom. 30 (1989) 465-503. MR 90g:53011

12. R. Kusner, R. Mazzeo \& D. Pollack, The moduli space of complete embedded constant mean curvature surfaces, Geom. and Funct. Analysis 1 (1996) 120-137. MR 97b:58022

13. R. Mazzeo \& D. Pollack, Gluing and moduli for noncompact geometric problems, preprint.

14. R. Mazzeo, D. Pollack \& K. Uhlenbeck, Moduli spaces of singular Yamabe metrics, J. Amer. Math. Soc. 2(9) (1996) 303-344. MR 96f:53055

15. W. H. Meeks III \& B. White, Minimal surfaces bounded by convex curves in parallel planes, Comment. Math. Helv. 66(2) (1991) 263-278. MR 92e:53013

16. J. Pérez, On singly-periodic minimal surfaces with planar ends, Trans. of the A.M.S. 349 (6) (1997) 2371-2389. MR 98b:53009

17. J. Pérez \& A. Ros, The space of properly embedded minimal surfaces with finite total curvature, Indiana Univ. Math. J. 451 (1996), 177-204. MR 97k:58034

18. J. Pérez \& A. Ros, One-ended minimal annuli bounded by a convex curve, in preparation. 
19. D. Pollack, Geometric moduli spaces as Lagrangian submanifolds, in preparation.

20. A. J. Tromba, Intrinsic third derivatives for Plateau's problem and the Morse inequalities for disc minimal surfaces in $\mathbb{R}^{3}$, Calc. Var. 1 (1993) 335-353. MR 95a:58024

21. M. Wohlgemuth, Higher genus minimal surfaces of finite total curvature, preprint.

Departamento de Geometria y Topologia, Facultad de Ciencias, Universidad de Granada, Fuentenueva, 18071, Granada, Spain

E-mail address: jperez@goliat.ugr.es

Departamento de Geometria y Topologia, Facultad de Ciencias, Universidad de Granada, Fuentenueva, 18071, Granada, Spain

E-mail address: aros@goliat.ugr.es 